\title{
Compensation and control of the birefringence of polymers for photonics
}

\author{
Akihiro Tagaya and Yasuhiro Koike \\ We demonstrated that a method based on the analysis of both orientational birefringence (intrinsic birefringence) and \\ photoelastic birefringence (photoelastic coefficient) is effective for designing polymers that are processed in a molten state and \\ used in a glassy state. The designed polymer exhibits no birefringence for any orientation of the polymer main chains or under \\ elastic deformation. Birefringence is close to zero even in injection-molded plates of the polymer. We have also shown that it \\ is possible to compensate and control birefringence of polymers using inorganic nanocrystals, and we have obtained valuable \\ information on the optimal size of nanocrystals for compensation and control of birefringence. Nanocrystals measuring \\ $\sim 200 \mathrm{~nm}$ in length and $20 \mathrm{~nm}$ in width are sufficiently small to compensate for birefringence, but sufficiently large to permit \\ control of orientation by casting. Reverse birefringence wavelength dispersion of a polymer film containing nanocrystals was \\ achieved by casting and heat drawing.
}

Polymer Journal (2012) 44, 306-314; doi:10.1038/pj.2011.141; published online 11 January 2012

Keywords: birefringence; liquid-crystal display; nanocrystals; orientational birefringence; photoelastic birefringence; photonics; zero-zero-birefringence polymer

\section{INTRODUCTION}

Owing to their ease of processing, low weight, high transparency and low cost, optical polymers are widely used as key materials for various optical devices, such as lenses, optical disks and optical films for liquid-crystal displays (LCDs). However, optical polymers frequently exhibit birefringence as a result of processing operations such as injection molding, extrusion or drawing, because polymer chains tend to become oriented when the polymer is in the molten state and oriented chains generally fail to relax completely during the cooling process. When a ray of light enters a birefringent medium, it is split into two rays, called the ordinary ray and the extraordinary ray, respectively. These rays propagate with different velocities in the birefringent medium because the refractive index for each ray is different. In most optical polymer devices, for example, optical films for LCDs, the rays combine with a certain phase difference as they emerge from the birefringent medium. As a result, the polarization state of the incident light is changed, for example, from linear polarization to elliptical polarization, which degrades the performance of optical devices that require maintenance of the polarization state of the incident light, such as LCDs. Birefringence can also degrade the performance of optical devices that require fine focusing by lenses, such as optical disk systems.

In the case of typical thermoplastic polymers used in optical polymer devices, the major type of birefringence is known as orienta- tional birefringence. This birefringence of polymers derives from the asymmetry of their molecular structures. Although most polymer chains have an intrinsic optical anisotropy derived from the asymmetry of their chemical structures, the bulk polymer material is macroscopically isotropic and exhibits no birefringence because its chains are randomly oriented and their intrinsic optical anisotropies cancel out one another (Figure 1). However, when the chains of a polymer are oriented, the material becomes birefringent because the intrinsic optical anisotropies of the chains do not then cancel each other out. This type of birefringence is called orientational birefringence. For measurements of orientational birefringence, we use polymer films that are uniaxially heat-drawn at a temperature that is above their glass-transition temperature $\left(T_{\mathrm{g}}\right)$ and subsequently cooled to room temperature. The resulting orientational birefringence is measured at room temperature (considerably below the $T_{\mathrm{g}}$ ), at which the polymer is in a glassy state. The $T_{\mathrm{g}}$ of thermoplastic polymers typically used for optical devices is generally above $90^{\circ} \mathrm{C}$. In the case of uniaxially heat-drawn polymer films, the orientational birefringence $\Delta n$ is defined as $\Delta n=n_{/ /}-n_{\perp}$, where $n_{/ /}$and $n_{\perp}$ are the refractive indices for light polarized in the directions parallel and perpendicular to the drawing (tensile stress) direction, respectively. Polymers with a positive birefringence show an orientational birefringence of $\Delta n>0$, and polymers with a negative birefringence have $\Delta n<0$. The sign of the birefringence is an inherent property of the polymer. The 


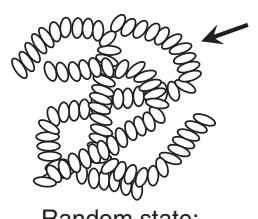

Random state:

Macroscopically isotropic
Polarizable ellipsoid of

a repeating unit

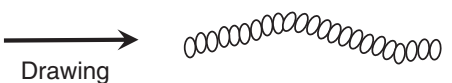

Oriented state:

Birefringence generated

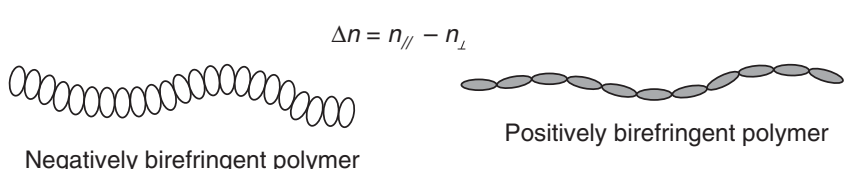

Figure 1 Mechanism for the generation of orientational birefringence.

relationship between the orientational birefringence $\Delta n$ and the degree of orientation of the polymer chains $f$ can be written as follows:

$$
\Delta n=f \times \Delta n^{0}
$$

Here, $\Delta n^{0}$ is the intrinsic birefringence, in other words, the orientational birefringence when the polymer chain is completely oriented $(f=1.0)$.

Research on the reduction of birefringence in optical polymer devices seems to have been actively pursued since the beginning of research and development on aspherical plastic lenses and disk substrates for optical disk systems. ${ }^{1-3}$ Recently, a great deal of research has been conducted on the reduction of birefringence in optical polymer films for LCDs. ${ }^{4}$ The research can be classified roughly into two categories. The first is based on approaches involving polymer processing, ${ }^{5-6}$ and the second is based on approaches involving the chemical design of polymers that exhibit a low birefringence. The aim of the former approach is to inhibit the orientation of polymer chains by adjusting the processing conditions, for example, by increasing the molding temperature and extending the annealing time for injection molding or by adopting processing methods such as a solvent casting. ${ }^{4}$ In the solvent-casting method, the polymer is dissolved in an organic solvent and the resulting solution is spread onto a flat and smooth substrate. Low-birefringence polymer films can be obtained by removing the organic solvent in a drying process. Although solvent casting is a practical method for manufacturing low-birefringence polymer films, large quantities of organic solvent are used and must be recovered, a process that requires a large investment in facilities. Therefore, melt extrusion, which requires no organic solvent and has the potential for high-speed production, is being adopted in industry. In melt extrusion, the polymer chains tend to become oriented, resulting in the generation of orientational birefringence, which limits the use of melt extrusion in the production of lowbirefringence optical films. At present, the manufacture of lowbirefringence polymer films is restricted to a few kinds of polymer. Furthermore, the rate of production of low-birefringence polymer films by melt extrusion is much lower than that of typical meltextrusion processes in industry.

The second approach, which involves the chemical design of polymers to exhibit a low birefringence, can be subdivided roughly into two categories. The first is based the design of polymers that have a high fluidity in their molten state. However, the reduction of birefringence of polymers to almost zero by this approach is difficult to achieve. The second involves the design of a polymer that is inherently almost optically isotropic. Such polymers are ideal for use in the optical devices mentioned above, because they exhibit almost no birefringence, even if their chains become oriented. Several
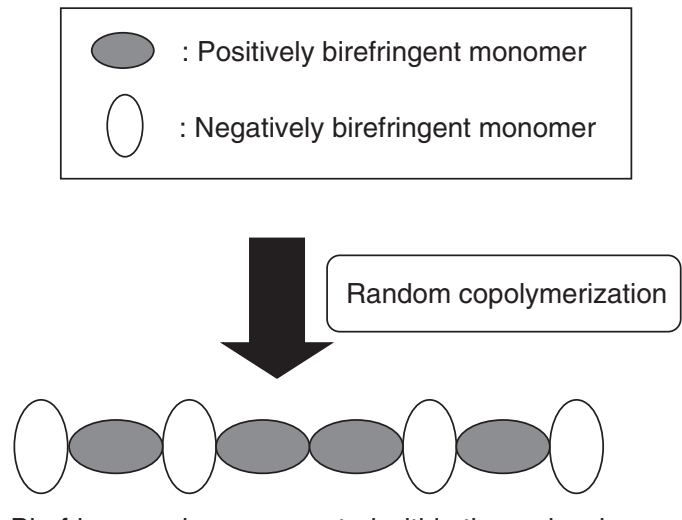

Birefringence is compensated within the molecule.

Figure 2 Concept of the random copolymerization method.

methods have been proposed for realizing optical polymers that exhibit no orientational birefringence for any orientation of their main chains. These include a random copolymerization, ${ }^{7-8}$ doping with an anisotropic molecule, ${ }^{9,10}$ doping with birefringent crystals ${ }^{11,12}$ or blending of polymers. ${ }^{13,14}$ In the random copolymerization method, a monomer of a homopolymer that shows a positive birefringence and a monomer of a homopolymer that shows negative birefringence are randomly copolymerized, as shown in Figure 2. As a result, there is a mutual canceling out of birefringence at a particular ratio of the two monomers. In the anisotropic molecule dopant method, the polymer is doped with a molecule that has an anisotropic polarizability, a rod-like shape, and a lower molecular weight than the polymer. When the polymer chains become oriented as a result of processes such as injection molding, extrusion or drawing, the molecules also become oriented as a result of their rod-like shape. A negative birefringence of the polymer can be compensated by doping with positively birefringent anisotropic molecules that have a higher polarizability in the direction of orientation than in the perpendicular direction.

The considerable research efforts discussed above have permitted the manufacture of low-birefringence pickup lenses, optical disks and optical films for LCDs, and have contributed to the widespread adoption, throughout the world, of instruments that use such optical devices. However, the reduction of the birefringence of polymers to an even lower level is becoming necessary because of the need to handle enormous volumes of data at higher speeds with high-performance instruments. For instance, high-resolution motion pictures are available from high-resolution video cameras for display on LCDs, and the resolution of these devices is continually increasing. As a result, photoelastic birefringence, which is observed in some types of optical polymer devices, has become a problem and its reduction has become important. For example, there are stringent requirements with regard to retardation films for LCDs that are required to possess a relatively high orientational birefringence but almost no photoelastic birefringence. A method for designing polymers that have the desirable birefringence properties is required to meet such requirements, and it should be established on the basis of research on the correlation between the birefringence properties and the chemical structures of polymers.

Here, we describe a method for designing polymers that exhibit no orientational birefringence and no photoelastic birefringence, which are potentially useful as components of devices for photonics applications. Furthermore, we describe techniques that use birefringent 
nanocrystals to expand the possibilities for compensation and control of the birefringence of polymers.

\section{ZERO-ZERO-BIREFRINGENCE POLYMERS}

Besides orientational birefringence, as described in the previous section, birefringence is generally observed in bulk polymers when they are deformed in glassy state. This type of birefringence is referred to as photoelastic birefringence, because most transparent polymers for optical polymer devices behave almost elastically at small deformations (less than $\sim 1 \%$ ). ${ }^{15}$ Figure 3 shows photographs of an injectionmolded plate of polycarbonate placed between crossed polarizers and illuminated from the rear by fluorescent lamps. When the plate was unstressed, the observed leakage of white light thorough the crossed polarizers was mainly the result of orientational birefringence (Figure 3a). When the plate was manually stressed, leakage of colored light occurred as a result of photoelastic birefringence (Figure $3 b$ ); this disappeared when the stress was released. The photoelastic birefringence $\Delta n$ is defined as follows:

$$
\begin{aligned}
& \Delta n=C \Delta \sigma \\
& \Delta \sigma=\sigma_{1}-\sigma_{2}
\end{aligned}
$$

where $C$ is the photoelastic coefficient of the polymer, $\sigma$ and $\sigma_{2}$ the principal stresses $\left(\sigma_{1}>\sigma_{2}\right)$ and $\Delta \sigma$ the difference between the two principal stresses. A positively birefringent polymer has a photoelastic coefficient $C>0$ and a negatively birefringent polymer has $C<0$. The principal stress is a tensile stress when its sign is positive and a compressive stress when its sign is negative. The sign of the photoelastic birefringence is defined in the same manner as the orientational

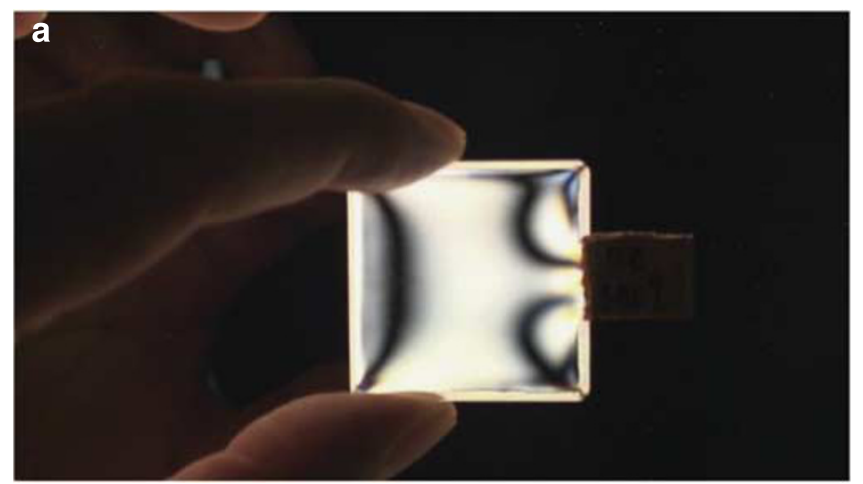

White light leakage mainly from orientational birefringence (Before adding stress).

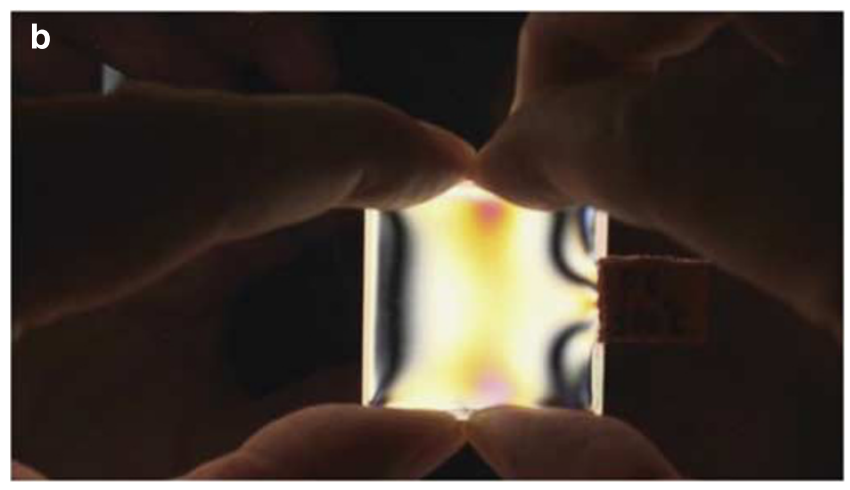

Colored light leakage due to photoelastic birefringence (During adding stress).

Figure 3 Photographs of an injection-molded polymer plate placed between crossed polarizers and illuminated by fluorescent lamps. (a) Before adding stress, (b) during adding stress. birefringence in the tensile-stress direction. The sign of orientational birefringence in some kinds of polymers, for example, polystyrene, is opposite to that of photoelastic birefringence, which shows that the change in the conformation of the polymer molecules that causes orientational birefringence is different from that which causes photoelastic birefringence. Although the behavior of optical polymer molecules in elastic deformation has not been clarified in detail, the orientation of the side chains of some kinds of methacrylates has been estimated to provide a major contribution to photoelastic birefringence compared with the orientation of the main chains of the polymer molecules subjected to an elastic deformation of $<1 \%{ }^{16}$ In the case of orientational birefringence, polymer main chains and side chains are significantly oriented above $T_{\mathrm{g}}$, and their orientations remain in the cooling process from the process temperature to room temperature.

In general, the volume of a polymer decreases when it is cooled from a temperature well above its $T_{\mathrm{g}}$ (its molten state) to room temperature (its glass state). In the case of injection molding or melt extrusion, photoelastic birefringence tends to be caused by the cooling process, particularly from around $T_{\mathrm{g}}$ to room temperature, because the polymer is solidified and stress accumulates. Therefore, a reduction in both orientational birefringence and photoelastic birefringence is required to produce low-birefringence polymer devices by means of injection molding or melt extrusion. ${ }^{17}$

Photoelastic birefringence can be eliminated by either random copolymerization method ${ }^{8}$ or by doping with an anisotropic molecule. ${ }^{15,18}$ The anisotropic molecules used in the anisotropic molecule dopant method become slightly oriented when side chains of polymer molecules are slightly oriented by elastic deformation of the optical polymer doped with anisotropic molecules. As a result, anisotropic molecules compensate for the photoelastic birefringence of the optical polymer.

In the case of the random copolymerization method, it should be pointed out that the composition of the copolymer that results in the elimination of orientational birefringence is different from that required to eliminate photoelastic birefringence. For example, in the case of copolymers of methyl methacrylate (MMA) and benzyl methacrylate (BzMA), the composition at which orientational birefringence is absent is MMA/BzMA $=82: 18$ (by mass), whereas that at which photoelastic birefringence is absent is MMA/BzMA $=92: 8$ (by mass). ${ }^{8}$ Therefore, it is not yet possible to simultaneously compensate for orientational birefringence and photoelastic birefringence by means of the random copolymerization method. Similarly, in the case of doping with an anisotropic molecule, the concentration of the anisotropic molecule required to eliminate orientational birefringence is different from that required to eliminate photoelastic birefringence. For example, the concentration of trans-stilbene $\left(1,1^{\prime}-(E)\right.$-ethene-1,2diyldibenzene) required to eliminate the orientational birefringence of poly(MMA) (PMMA) is $3.0 \mathrm{wt} \%,{ }^{9}$ whereas that required to eliminate photoelastic birefringence of the same polymer is $2.2 \mathrm{wt} \%{ }^{18}$ Therefore, simultaneous compensation of orientational and photoelastic birefringence by doping with an anisotropic molecule has also yet to be demonstrated.

We define polymers that exhibit no birefringence for any degree of orientation of the polymer main chains or when subjected to elastic deformation (that is, for which both orientational and photoelastic birefringence are zero) as zero-zero-birefringence polymers. We have demonstrated that zero-zero-birefringence polymers can be produced in systems consisting of more than three components, in which at least one of the components exhibits the opposite orientational birefringence effect and the opposite photoelastic birefringence effect to those of the other components. ${ }^{19}$ The design of zero-zero-birefringence 
polymers is based on an analysis of the intrinsic birefringence and the photoelastic coefficient of various copolymers, as described below.

Ternary copolymers were synthesized from MMA, 2,2,2-trifluoroethyl methacrylate (3FMA) and BzMA. The signs of the birefringence effect of these monomers are listed in Table $1 .^{19}$ The binary copolymers poly(MMA-co-3FMA) and poly(MMA-co-BzMA) were synthesized in a range of compositions as a preliminary stage in the design of a ternary copolymer. Figure 4 shows the intrinsic birefringence and the

Table 1 Signs of the birefringence effects of the various monomers

\begin{tabular}{lll}
\hline Monomer & Orientational birefringence & Photoelastic birefringence \\
\hline MMA & Negative & Negative \\
3FMA & Positive & Negative \\
BzMA & Positive & Positive
\end{tabular}

Abbreviations: BzMA, benzyl methacrylate; MMA, methyl methacrylate; 3FMA, 2,2,2trifluoroethyl methacrylate.
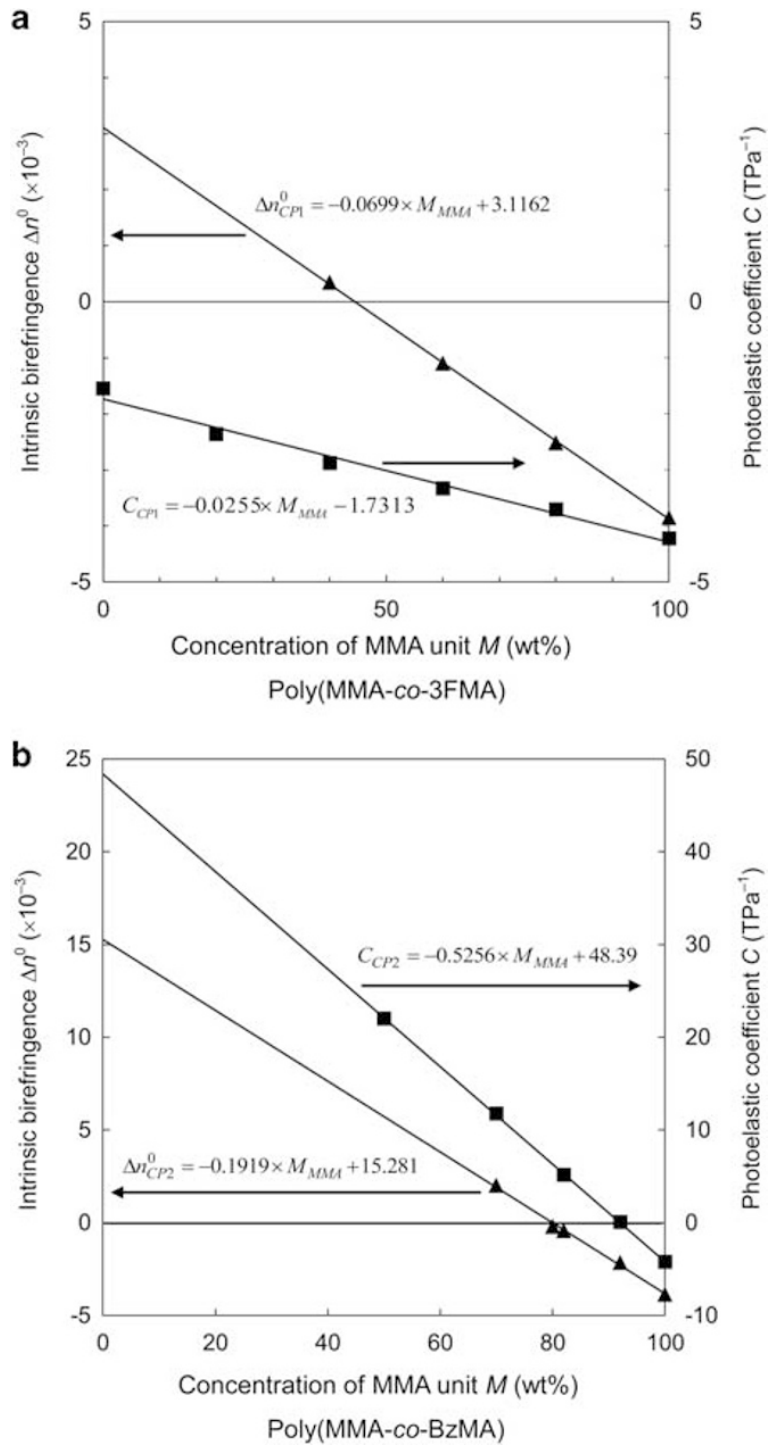

Figure 4 Intrinsic birefringence and photoelastic coefficient as a function of the composition for (a) poly(MMA-co-3FMA) and (b) poly(MMA-co-BzMA). BzMA, benzyl methacrylate; MMA, methyl methacrylate; 3FMA, 2,2,2trifluoroethyl methacrylate. photoelastic coefficients of the resulting binary copolymers in terms of the concentration of MMA units that are present. The relations between these coefficients can be described by the approximate equations (4)-(7).

$$
\begin{aligned}
& \Delta n_{\mathrm{CP} 1}^{0}=-0.0699 \times M_{\mathrm{MMA}}+3.1162 \\
& C_{\mathrm{CP} 1}=-0.0255 \times M_{\mathrm{MMA}}-1.7313 \\
& \Delta n_{\mathrm{CP} 2}^{0}=-0.1919 \times M_{\mathrm{MMA}}+15.281 \\
& C_{\mathrm{CP} 2}=-0.5256 \times M_{\mathrm{MMA}}+48.39
\end{aligned}
$$

Here, $\Delta n_{\mathrm{CP} 1}^{0}\left[\times 10^{-3}\right]$ and $\mathrm{C}_{\mathrm{CP} 1}\left[\mathrm{TPa}^{-1}\right]$ are, the intrinsic birefringence and the photoelastic coefficient respectively, for poly(MMA-co3FMA), whereas $\Delta n_{\mathrm{CP} 2}^{0}\left[\times 10^{-3}\right]$ and $\mathrm{C}_{\mathrm{CP} 2}\left[\mathrm{TPa}^{-1}\right]$ are, the intrinsic birefringence and the photoelastic coefficient respectively, for poly (MMA-co-BzMA). $M_{\mathrm{MMA}}$ [wt\%] is the concentration of MMA units in poly(MMA-co-3FMA) or poly(MMA-co-BzMA).

The contributions of MMA to the intrinsic birefringence and the photoelastic coefficient are $\Delta n_{\mathrm{PMMA}}^{0}=-3.8738\left[\times 10^{-3}\right]$ and $C_{\mathrm{PMMA}}=-4.2813\left[\mathrm{TPa}^{-1}\right]$, which are obtained when $M_{\mathrm{MMA}}=100$ wt $\%$ in equations (4) and (5), respectively. In addition, the contributions of 3FMA to the intrinsic birefringence and the photoelastic coefficient are $\Delta n_{\mathrm{P} 3 \mathrm{FMA}}^{0}=3.1378\left[\times 10^{-3}\right]$ and $C_{\mathrm{P} 3 \mathrm{FMA}}=-1.7313$ $\left[\mathrm{TPa}^{-1}\right]$, which are obtained when $C_{\mathrm{M} 1}=0 \mathrm{wt} \%$ in Equations (4) and (5), respectively. Similarly, the contributions of BzMA to the intrinsic birefringence and the photoelastic coefficient are $\Delta n_{\mathrm{PBzMA}}^{0}=15.281\left[\times 10^{-3}\right]$ and $C_{\mathrm{PbzMA}}=48.39\left[\mathrm{TPa}^{-1}\right]$, which are obtained when $C_{\mathrm{M} 1}=0 \mathrm{wt} \%$ in equations (6) and (7), respectively.

For a terpolymer of MMA, 3FMA and BzMA, the weight fractions of the monomers can be written as $\alpha$ (wt $\%), \beta$ (wt $\%)$ and $\gamma$ (wt $\%$ ), and

$$
\alpha+\beta+\gamma=100
$$

On the basis of the contributions discussed above, the intrinsic birefringence $\Delta n^{0}\left[\times 10^{-3}\right]$ and the photoelastic coefficient $C\left[\mathrm{TPa}^{-1}\right]$ for the terpolymer poly(MMA-co-3FMA-co-BzMA) can be written as

$$
\begin{aligned}
\Delta n^{0} & =\Delta n_{\mathrm{PMMA}}^{0} \times \frac{\alpha}{100}+\Delta n_{\mathrm{P} 3 \mathrm{FMA}}^{0} \times \frac{\beta}{100}+\Delta n_{\mathrm{PBzMA}}^{0} \times \frac{\gamma}{100} \\
& =-3.7838 \times \frac{\alpha}{100}+3.1378 \times \frac{\beta}{100}+15.281 \times \frac{\gamma}{100} \\
C & =C_{\mathrm{PMMA}} \times \frac{\alpha}{100}+C_{\mathrm{P} 3 \mathrm{FMA}} \times \frac{\beta}{100}+C_{\mathrm{PBzMA}} \times \frac{\gamma}{100} \\
& =-4.2813 \times \frac{\alpha}{100}-1.7313 \times \frac{\beta}{100}+48.39 \times \frac{\gamma}{100}
\end{aligned}
$$

By solving equations (8)-(10) simultaneously for the condition that $\Delta n^{0}=C=0$, we obtained the specified composition MMA/3FMA/ $\mathrm{BzMA}=55.5: 38.0: 6.5$ (by mass). We synthesized a sample of poly (MMA-co-3FMA-co-BzMA) with this composition, and we measured its orientational birefringence and photoelastic birefringence at a wavelength of $633 \mathrm{~nm}$ by optical heterodyne interferometry using birefringence measurement equipment (Uniopt Corporation, Shizuoka, Japan, ABR-10A). The results are shown in Figure 5 and Table $2 .{ }^{19}$ Poly (MMA-co-3F-c-Bz 3FMA-co-BzMA) of the specific composition exhibited a very low orientational birefringence and a very low photoelastic birefringence; these are negligible amounts of birefringence for a typical optical device. By fine adjustment of the composition to MMA/3FMA/ $\mathrm{BzMA}=52.0: 42.0: 6.0$ (by mass), we obtained an optical polymer that exhibited almost no birefringence. ${ }^{19}$ No birefringence at wavelengths of 
a
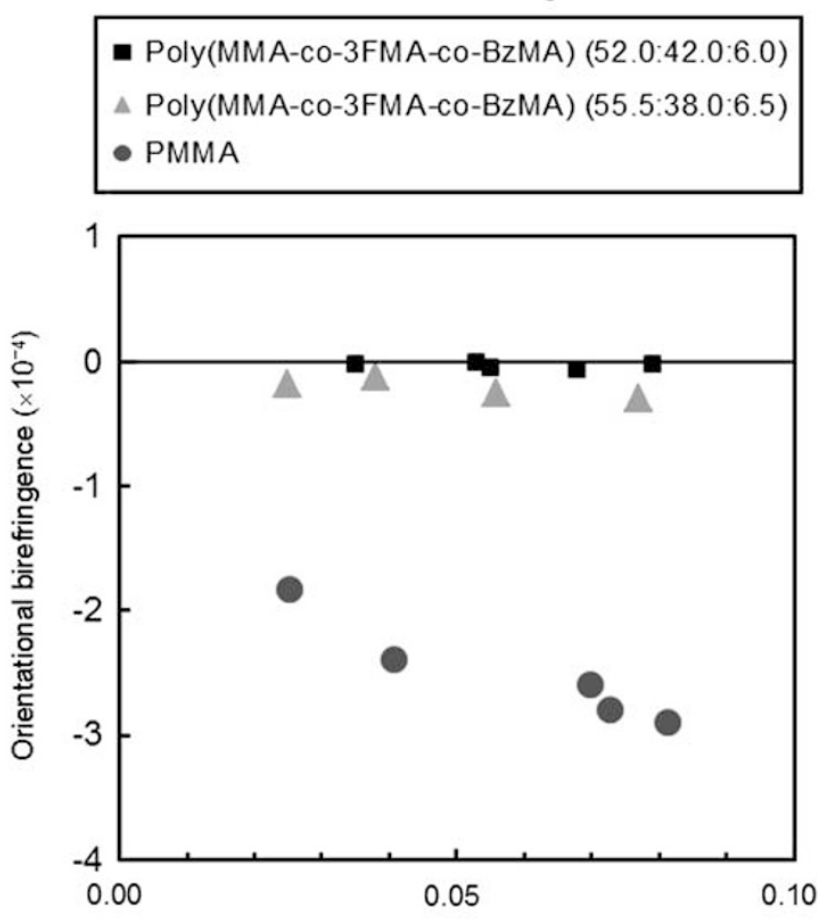

Orientation degree of main chains of the polymers

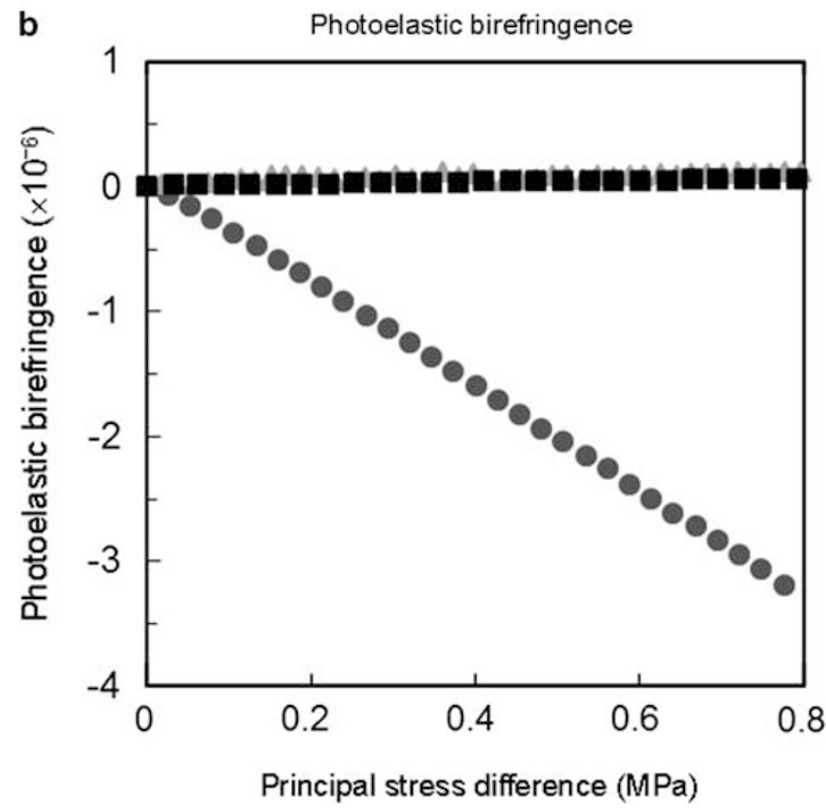

Figure 5 Orientational birefringence and photoelastic birefringence of a ternary copolymer compared with PMMA: (a) orientational birefringence as a function of the degree of orientation of the main chains of poly(MMA-co3FMA-co-BzMA); (b) photoelastic birefringence as a function of the principal stress difference in poly(MMA-co-3FMA-co-BzMA). Reprinted with permission from the study by Tagaya et al. Copyright 2006 American Chemical Society. BzMA, benzyl methacrylate; MMA, methyl methacrylate; 3FMA, 2,2,2-trifluoroethyl methacrylate; PMMA, poly(MMA). A full color version of this figure is available at Polymer Journal online.
Table 2 Photoelastic coefficients of ternary copolymers

\begin{tabular}{lc}
\hline Optical polymer & Photoelastic coefficient $\left(\mathrm{Pa}^{-1}\right)$ \\
\hline $\begin{array}{l}\text { poly(MMA-co-3FMA-co-BzMA } \\
(55.5: 38.0: 6.5)\end{array}$ & $0.12 \times 10^{-12}$ \\
& \\
poly(MMA-co-3FMA-co-BzMA) & \\
$(52.0: 42.0: 6.0)$ & $0.07 \times 10^{-12}$ \\
PMMA & $-4.23 \times 10^{-12}$ \\
\hline
\end{tabular}

Abbreviations: BzMA, benzyl methacrylate; MMA, methyl methacrylate; 3FMA, 2,2,2trifluoroethyl methacrylate; PMMA, poly(MMA)

450.1, 497.2, 548.9, 585.8, 627.8 and $752.4 \mathrm{~nm}$ was detected by parallel Nicol method using birefringence measurement equipment (Oji Scientific Instruments, Hyogo, Japan, KOBRA-WPR), which means wavelength dispersion of birefringence of the polymer is almost flat.

As described above, the zero-zero-birefringence polymer was designed and synthesized on the basis of analyses of the orientational birefringence (intrinsic birefringence) present in uniaxially heat-drawn polymer films and the photoelastic birefringence (photoelastic coefficient) caused by elastic deformation of the bulk polymers. We also analyzed birefringence produced in injection-molded plates of the polymers to evaluate the effectiveness of our design method in injection molding. We found that orientational birefringence and photoelastic birefringence were almost zero in injection-molded plates and they did not depend on the molding temperature. ${ }^{17}$ We therefore conclude that we have demonstrated the effectiveness of our design method and that our ternary polymer is an ideal zero-zero-birefringence polymer.

Recently, a polarized laser backlight was produced using an injection-molded light-guide plate made from our zero-zero-birefringence polymer. ${ }^{20} \mathrm{~A}$ laser was converted into an area light source by reflection and refraction at the light guide plate. The state of polarization of the incident laser beam was maintained even after it had propagated through the light-guide plate, because the birefringence of the lightguide plate was almost zero. The polarized laser backlight is a promising candidate for use as the next generation of backlights for LCDs, because it has considerable potential for improving the efficiency and color gamut of the display.

Clearly, this design method can be applied to other polymers composed of other monomers, opening the way to the synthesis of novel zero-zero-birefringence polymers. The design of polymers containing more than three types of monomer unit is described below. Using the methods shown above, it is possible to obtain three empirical equations related to the intrinsic birefringence (such as equation (9)), the photoelastic coefficient (such as equation (10)) and the composition (such as equation (8)). Therefore, if the number of components is increased beyond three, it is not possible to solve the equations analytically. In the case of optical polymers, the number of the components is generally not more than 10 and is typically fewer than 5. For most real cases, it is therefore possible to solve the equations numerically by using a computer. There could be several solutions for a polymer composed of more than three components, and there might be no solution for a polymer composed of inappropriate components. The number of solutions tends to increase as the number of components increases.

Figure 6 shows a plot of the photoelastic coefficient against the intrinsic birefringence. We call this type of plot a birefringence map. Plots for PMMA, P3FMA and PBzMA (corresponding to the monomers of the zero-zero-birefringence polymer) form a triangle that 


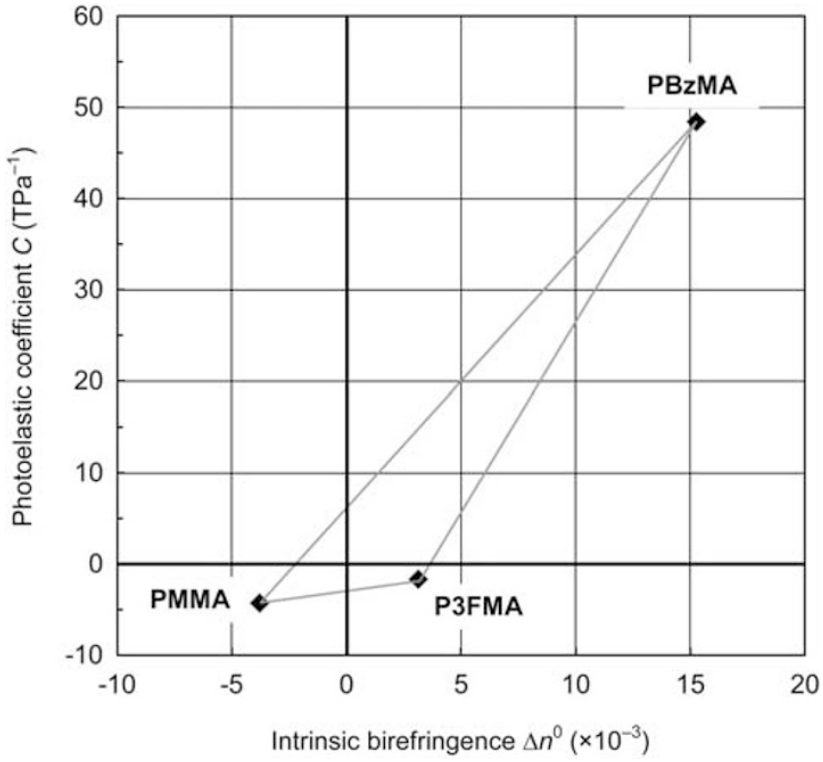

Figure 6 Birefringence properties of PMMA, P3FMA and PBzMA plotted as a birefringence map. BzMA, benzyl methacrylate; 3FMA, 2,2,2-trifluoroethyl methacrylate; PMMA, poly(methyl methacrylate). A full color version of this figure is available at Polymer Journal online.

contains the origin, which means that the composition for the zerozero-birefringence polymer can be obtained by solving equations (8)(10) with the condition that $\Delta n^{0}=C=0$. The birefringence map is a powerful tool for identifying suitable monomers for zero-zero-birefringence polymers, because promising monomers can be identified visually. Analyses of intrinsic birefringences and photoelastic coefficients are still proceeding with the aim of adding points to the birefringence map.

Polymers having desirable birefringence properties can also be designed in a similar manner by applying our method, because the appropriate composition can be obtained by solving the equations simultaneously with the appropriate set values for the intrinsic birefringence and photoelastic coefficient. Polymers having a relatively high intrinsic birefringence and almost no photoelastic coefficient are suitable for use as retardation films, which are used to provide wide viewing angles for LCDs. Previously, there was a tendency to use molecular design to decrease optical anisotropy for reducing orientational birefringence or photoelastic birefringence, because the mechanisms responsible for orientational birefringence and photoelastic birefringence had not been clarified in detail. As a result, the independent control of these types of birefringence by means of molecular design was difficult to achieve. Although the elucidation of the mechanisms responsible for orientational birefringence and photoelastic birefringence is ongoing, polymers having desirable birefringence properties can be quantitatively designed by our method.

\section{COMPENSATION AND CONTROL OF BIREFRINGENCE BY A BIREFRINGENT CRYSTAL}

As mentioned above, the random copolymerization method, the anisotropic dopant method and the polymer blending method can all be used to control the birefringence of polymers. All these methods use organic compounds exclusively and they are based on the concept of compensation through mixing of materials that display positive and negative birefringence. The use of inorganic compounds to compensate for birefringence in polymers could markedly expand the range of design possibilities in the compensation of birefringence of polymers. a

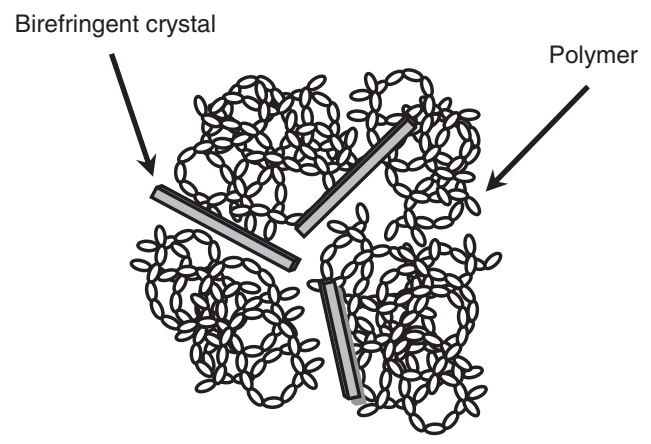

Amorphous state

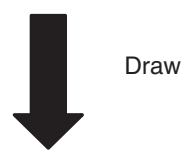

b

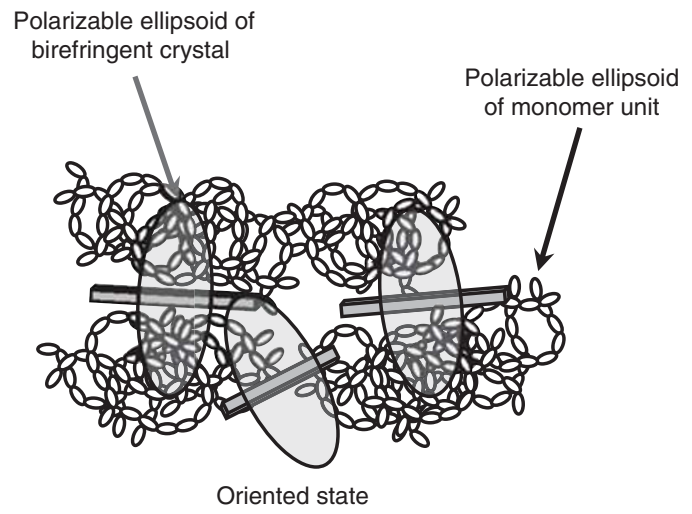

Figure 7 Concept of the birefringent crystal dopant method. (a) Amorphous state, (b) oriented state. A full color version of this figure is available at Polymer Journal online.

The anisotropic dopant method has the advantage that the negative birefringence of widely used polymers can be compensated merely by adding small amounts of anisotropic molecules to the polymers. However, no anisotropic molecules that exhibit relatively high negative birefringence effects are currently available. Molecules for the anisotropic dopant method need to have a rod-like shape and a high anisotropy of polarizability, such as trans-stilbene. Anisotropic molecules generally contain a conjugated double-bond system along their long axis to produce their rod-like shape, which makes polarizability along the long axis the highest. As a result, anisotropic molecules exhibit a positive birefringence when they become oriented in the same direction as the polymer chains, when the latter are oriented. It is therefore difficult to design anisotropic organic molecules that exhibit a high negative birefringence. However, it is well known that needlelike crystals of some inorganic compounds, such as calcium carbonate or strontium carbonate, show a large birefringence, and it has been shown that these can be used as birefringence-controlling dopants. ${ }^{11,12,21}$ In this method, needle-like nanocrystals are added to polymers and they become oriented when the polymer chains are oriented, as shown in Figure 7. As a result, birefringence of the polymer is compensated by birefringence of the crystals. Before the first demonstration by the birefringent crystal dopant method, the most important point to consider was the size of the birefringent 
crystal dopant. On the basis of results obtained using anisotropic organic molecule dopants, it was possible to conclude that birefringence of a polymer can be compensated by doping with birefringent crystals that have similar dimensions to those of the anisotropic organic molecule dopants. However, it is difficult to synthesize small birefringent crystals with a needle-like shape. Therefore, we needed to know what is the maximum size of units with positive and negative optical anisotropies that when randomly mixed form an optically isotropic medium. We could find no clear answer to this question in the standard textbooks on optics; however, it appears to be less than the size of an optical wavelength.

We successfully demonstrated compensation for the positive birefringence of poly(MMA-co-BzMA) (78:22 by mass) by doping with crystals of strontium carbonate $\left(\mathrm{SrCO}_{3}\right) \cdot{ }^{11-12}$ The refractive index for light polarized along the long axis of the needle-like $\mathrm{SrCO}_{3}$ crystal is 1.5199, whereas those along the other axes are 1.666 and 1.6685 . The $\mathrm{SrCO}_{3}$ crystals were synthesized by injecting $\mathrm{CO}_{2}$ gas into an aqueous suspension of $\mathrm{Sr}(\mathrm{OH})_{2}$. The crystals had a rod-like shape with a length of $\sim 200 \mathrm{~nm}$ and a width of $\sim 20 \mathrm{~nm}$. The crystals were surface treated with bis(dioctyl pyrophosphate)oxyacetate titanate-coupling agent and added to a solution of poly(MMA-co-BzMA) in tetrahydrofuran, and a film was prepared by casting from solution. The thickness of the polymer film was $30 \mu \mathrm{m}$. Figure 8 shows photographs of the poly(MMA-co-BzMA) films placed between crossed polarizers and illuminated from behind by fluorescent lamps. ${ }^{11}$ The unoriented poly(MMA-co-BzMA) film exhibited no birefringence, as shown in Figure $8 \mathrm{a}$. The polarization state of the transmitted light was almost undisturbed by poly(MMA-co-BzMA) films containing $0.5 \mathrm{wt} \%$ or 1.0 wt $\%$ of $\mathrm{SrCO}_{3}$ (Figures $8 \mathrm{~b}$ and c), respectively. In contrast, a film containing large $\mathrm{SrCO}_{3}$ crystals shows birefringence and depolarized scattering (Figure $8 \mathrm{~d}$ )]. The $3.0 \mu \mathrm{m} \times 300 \mathrm{~nm}$ crystals are too large to form an optically isotropic mixture with the polymer. As a result, the light transmitted through the birefringent crystals and that transmitted through the polymer around the birefringent crystals become independent of one another.

The orientational birefringence of the poly(MMA-co-BzMA) film containing 200-nm-long $\mathrm{SrCO}_{3}$ nanocrystals and uniaxially heatdrawn at $130{ }^{\circ} \mathrm{C}$ is shown in Figure 9. ${ }^{11}$ The poly(MMA-co-BzMA) film containing $0.4 \mathrm{wt} \%$ of 200 -nm-long $\mathrm{SrCO}_{3}$ nanocrystals showed a negative orientational birefringence, whereas the undoped poly
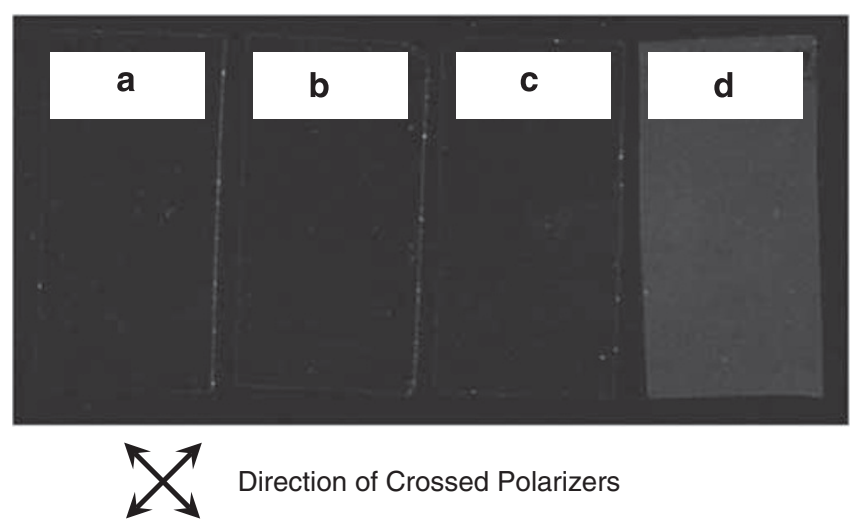

Direction of Crossed Polarizers

Figure 8 Photograph of various polymer films placed between crossed polarizers. (a) poly(MMA-co-BzMA), (b) poly(MMA-co-BzMA) containing 0.5 wt\% 200-nm-long, 20-nm-wide $\mathrm{SrCO}_{3}$ crystals, (c) poly(MMA-co-BzMA) containing 1.0 wt\% 200-nm-long, 20-nm-wide $\mathrm{SrCO}_{3}$ crystals and (d) containing $0.5 \mathrm{wt} \% 3.0-\mu \mathrm{m}$-long, 300-nm-wide $\mathrm{SrCO}_{3}$ crystals. This figure is cited from the study by Tagaya et al. ${ }^{11}$ BzMA, benzyl methacrylate; MMA, methyl methacrylate.
(MMA-co-BzMA) film showed a positive orientational birefringence. The negative value of the orientational birefringence increased with increasing crystal concentration of the nanocrystals. The orientational birefringence of poly(MMA-co-BzMA) was almost eliminated by doping with $0.3 \mathrm{wt} \%$ of $\mathrm{SrCO}_{3}$ for draw ratios in the region 1.0-2.0. The orientation of the needle-like $\mathrm{SrCO}_{3}$ crystal particles in the drawing direction was confirmed by transmission electron microscopy. Therefore, we concluded that the orientational birefringence of the polymer was compensated by doping with birefringent crystals. This birefringent crystal dopant method is applicable to other polymers, and other kinds of birefringent crystals are potential candidates for use as dopants.

In addition to the compensation of birefringence, the birefringent crystal dopant method was applied to control the birefringence and its wavelength dispersion of polymer films, because these properties are important for retardation films used in LCDs. ${ }^{22}$ Although the absolute value of the birefringence of polymers generally decreases with increasing wavelength, retardation films with a reverse birefringence dispersion in which the absolute value of the birefringence increases with increasing wavelength are required. Some types of retardation films prepared by mixing various components have been reported. ${ }^{23,24}$ However, before the demonstration of the birefringent crystal dopant method, selection of the components was limited to organic compounds. $\mathrm{SrCO}_{3}$ nanocrystals can be oriented by casting, which opens the way to the design of a range of retardation films. For design of retardation films, birefringence wavelength dispersion of the $\mathrm{SrCO}_{3}$ nanocrystals was measured in uniaxially heat-drawn poly(MMA-coBzMA) films that exhibit no orientational birefringence. Figure 10 shows the design concept for a polymer film with a reverse dispersion prepared by doping the polymer with $\mathrm{SrCO}_{3}$ crystals, solvent casting to form a film and then drawing the heated film. ${ }^{22}$ By this method, a cycloolefin copolymer film containing $\mathrm{SrCO}_{3}$ nanocrystals that exhibited reverse dispersion was fabricated, as shown in Figure 10. The orientation of the $\mathrm{SrCO}_{3}$ nanocrystals was confirmed by means of transmission electron microscopy. The nanocrystals were oriented immediately after casting, and then the orientation was enhanced by uniaxial heat drawing (Figure 11). ${ }^{22}$ Polymer chains were also oriented by uniaxial heat drawing. Therefore, the ratio the degree of orientation

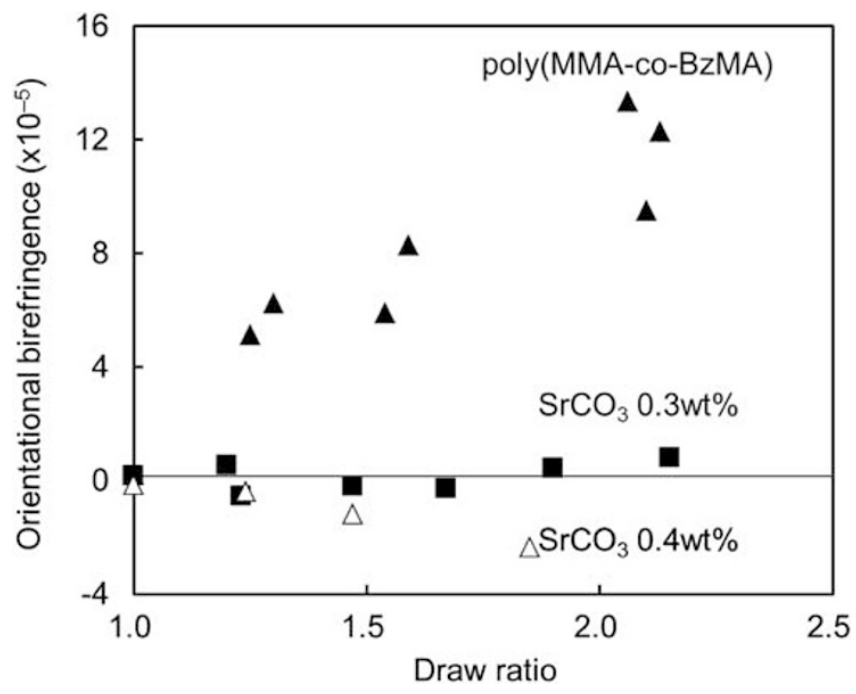

Figure 9 Orientational birefringence of the poly(MMA-co-BzMA) films containing $\mathrm{SrCO}_{3}$ with respect to the draw ratio. This figure is cited from the study by Tagaya et al. ${ }^{11}$ BzMA, benzyl methacrylate; MMA, methyl methacrylate; 3FMA, 2,2,2-trifluoroethyl methacrylate. 


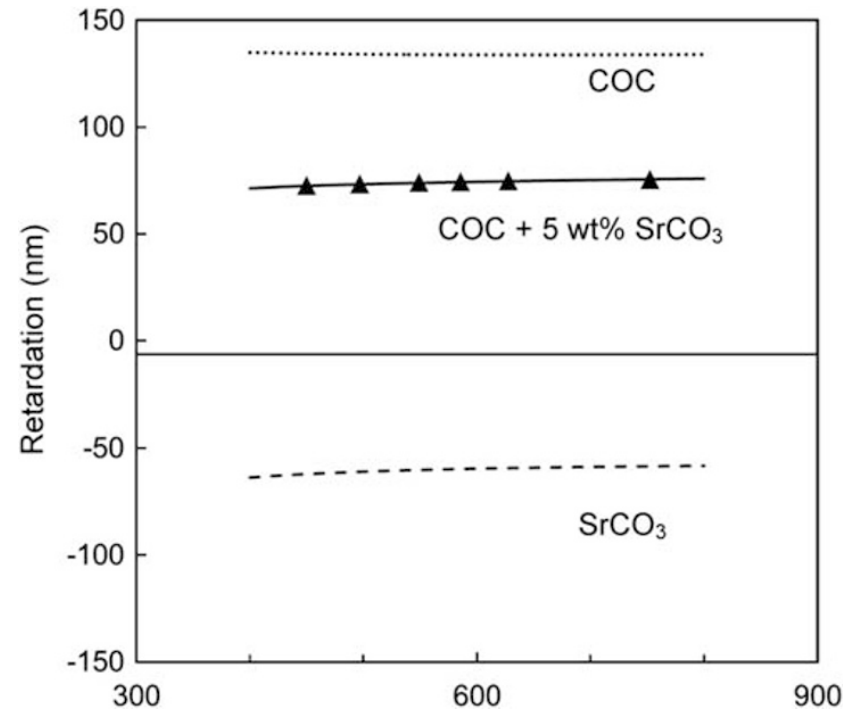

Figure 10 The retardation of a $55-\mu \mathrm{m}$-thick cycloolefin copolymer (COC) film doped with $5 \mathrm{wt} \% \mathrm{SrCO}_{3}$, plotted against the wavelength. The orientation function of $\mathrm{SrCO}_{3} f_{\mathrm{SrCO}}=0.42$. Reprinted with permission from the study by Shafiee et al.22 Copyright 2011 The Japan Society of Applied Physics.

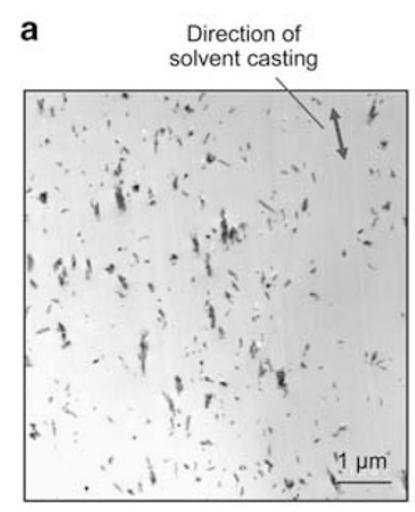

After casting

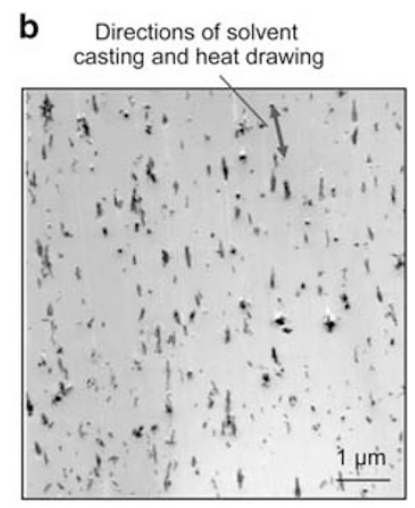

After casting and heat drawing
Figure 11 Transmission electron micrographs $(\times 20000)$ of a cycloolefin copolymer (COC) film containing 5 wt $\% \mathrm{SrCO}_{3}$ crystals. (a) After casting. (b) After casting and heat drawing. Reprinted with permission from the study by Shafiee et al. Copyright 2011 The Japan Society of Applied Physics. A full color version of this figure is available at Polymer Journal online.

degrees of the nanocrystals to that of the polymer chains can be controlled by adjusting the conditions for the casting and for the uniaxial heat drawing. Furthermore, the direction of orientation of the nanocrystals can be freely controlled because the casting direction is independent of that of the uniaxial heat drawing. As shown in Figure 12, the transmittance of the cycloolefin copolymer film containing $5 \mathrm{wt} \% \mathrm{SrCO}_{3}$ crystals was $89.1 \%$ at the wavelength of $550 \mathrm{~nm}$, whereas that of the undoped film was $90.7 \%$ at the same wavelength. ${ }^{22}$

Poly(MMA/BzMA=92/8 (wt)) film doped with $5.0 \mathrm{wt} \%$ of $\mathrm{SrCO}_{3}$ crystals was prepared to investigate the effect of the $\mathrm{SrCO}_{3}$ crystals on photoelastic birefringence. ${ }^{25}$ The photoelastic coefficient of the polymer film was about $-1.1 \times 10^{-12} \mathrm{~Pa}^{-1}$. The photoelastic coefficient by the $5.0 \mathrm{wt} \%$ of $\mathrm{SrCO}_{3}$ crystals estimated to be about $-0.9 \times 10^{-12} \mathrm{~Pa}^{-1}$ because the poly(MMA/BzMA $=92 / 8 \quad(w t))$ film exhibited $-0.2 \times 10^{-12} \mathrm{~Pa}^{-1}$.

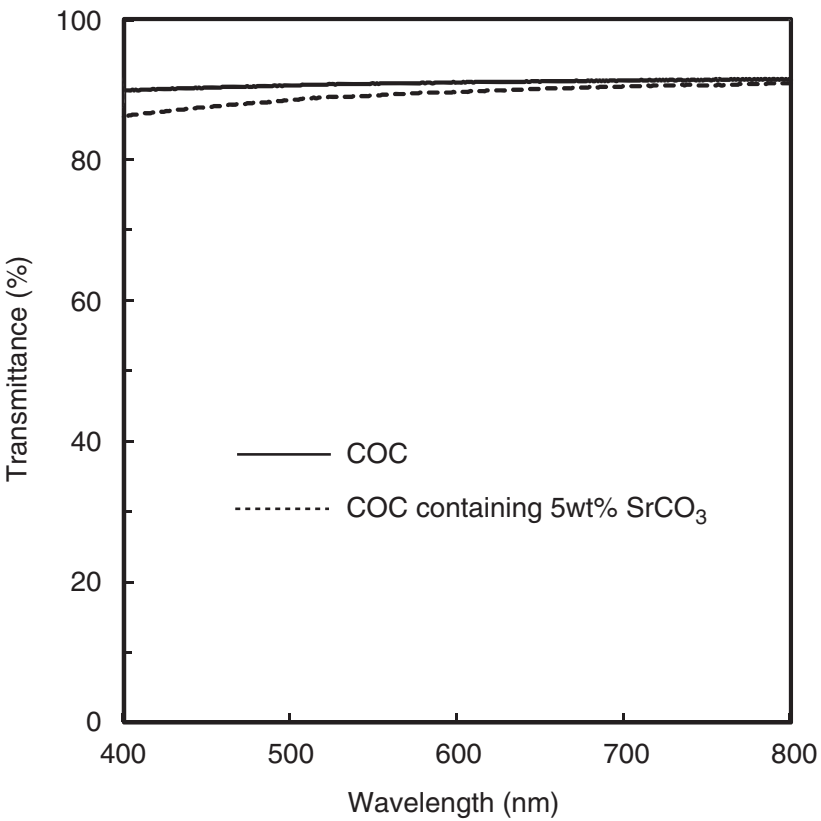

Figure 12 Transmittance of $\mathrm{COC}$ film containing $5 \mathrm{wt} \% \mathrm{SrCO}_{3}$ crystals and an undoped $\mathrm{COC}$ film. The thickness $t=55 \mu \mathrm{m}$. Reprinted with permission from the study by Shafiee et al. Copyright 2011 The Japan Society of Applied Physics. COC, cycloolefin copolymer.

\section{CONCLUSIONS}

The ability to compensate and control the birefringence of polymers has progressed from the stage of merely mixing positive and negative components to that of quantitative design based on analyses of orientational birefringence and photoelastic birefringence. A considerable amount of research has been performed on the birefringence of polymers, in which birefringence of polymers has been analyzed not only in the glassy state but also in the molten and solution states. Stress-induced conformational changes in polymers depend on the physical states of the material, a fact that should stimulate research on the analysis of relationships between birefringence and stress or other factors. In the case of transparent thermoplastic polymers for optical devices, before demonstration of the zero-zero-birefringence polymer, it had not been obvious which type of birefringence should be analyzed in relation to molecular design to reduce and control birefringence. We showed that a design method based on the analysis of both orientational birefringence (intrinsic birefringence) and photoelastic birefringence (the photoelastic coefficient) is effective for polymers processed in the molten state and used in the glassy state. In addition, we described the first compensation and control of birefringence of polymers by means of inorganic nanocrystals. The size of the nanocrystals (a length of $\sim 200 \mathrm{~nm}$ and a width of $\sim 20 \mathrm{~nm}$ ) was sufficiently small to permit compensation for birefringence, but sufficiently large to permit control of orientation by casting.

\section{CONFLICT OF INTEREST}

The authors declare no conflict of interest.

\section{ACKNOWLEDGEMENTS}

Some of this research was supported by the Japan Society for the Promotion of Science (JSPS) through its 'Funding Program for World-Leading Innovative R\&D on Science and Technology' (FIRST Program). 
1 Zwiers, R. J. M. \& Dortant, G. C. M. Aspherical lenses produced by a fast high-precision replication process using UV-curable coatings. Appl. Opt. 24, 4483-4488 (1985).

2 Kawai, H., Suzuki, M. \& Yoshida, A. Birefringence-free acrylic resin for precision plastic optics. Proc. SPIE 3135, 42-51 (1997).

3 Takahashi, A., Mieda, M., Murakami, Y., Ohta, K. \& Yamaoka, H. Influence of birefringence on the signal quality of magnetooptic disks using polycarbonate substrates. Appl. Opt. 27, 2863-2866 (1988).

4 Sata, H., Masahiko, M. \& Shimamoto, S. Properties and applications of cellulose triacetate film. Macromol. Symp. 208, 323-333 (2004).

5 Chen, Y. C., Chen, C. H. \& Chen, S. C. Effects of processing conditions on birefringence development in injection molded parts. II. Experimental measurement. Polym. Int. 40, 251-259 (1996).

6 Maruyama, T., Takagi, M., Noro, Y., Masuda, M. \& Ohtsu, H. Zoom lens systems with aspherical plastic lens. IEEE Trans. Consumer Electr. CE-33, 256-266 (1987).

7 Iwata, S., Tsukahara, H., Nihei, E. \& Koike, Y. Compensation for birefringence of oriented polymers by random copolymerization method. Jpn. J. Appl. Phys. 35, 3896-3901 (1996).

8 Iwata, S., Tsukahara, H., Nihei, E. \& Koike, Y. Transparent zero-birefringence copolymer and its optical properties. Appl. Opt. 36, 4549-4555 (1997).

9 Tagaya, A., Iwata, S., Kawanami, E., Tsukahara, H. \& Koike, Y. Zero-birefringence polymer by the anisotropic molecule dope method. Appl. Opt. 40, 3677-3683 (2001).

10 Tagaya, A., Iwata, S., Kawanami, E., Tsukahara, H. \& Koike, Y. Anisotropic molecule dopant method for synthesizing a zero-birefringence polymer. Jpn. J. Appl. Phys. 40, 6117-6123 (2001).

11 Tagaya, A., Ohkita, H., Mukoh, M., Sakaguchi, R. \& Koike, Y. Compensation of the birefringence of a polymer by a birefringent crystal. Science (Washington, DC) $\mathbf{3 0 1}$, 812-814 (2003).

12 Ohkita, H., Tagaya, A. \& Koike, Y. Preparation of a zero-birefringence polymer doped with a birefringent crystal and analysis of its characteristics. Macromolecules 37, 8342-8348 (2004)

13 Hahn, B. R. \& Wendorff, J. H. Compensation method for zero birefringence in oriented polymers. Polymer 26, 1619 (1985)
14 Saito, H., \& Inoue, T. Chain orientation and intrinsic anisotropy in birefringence-free polymer blends. J. Polym. Sci., Part B: Polym. Phys. 25, 1629-1636 (1987).

15 Ohkita, H., Ishibashi, K., Tanaka, R., Tagaya, A. \& Koike, Y. Compensation of the photoelastic birefringence of polymers by anisotropic molecules and analysis of the compensation mechanism. Jpn. J. Appl. Phys. 44, 3975-3982 (2005).

16 Shafiee, H., Tagaya, A. \& Koike, Y. Mechanism of generation of photoelastic birefringence in methacrylate polymers for optical devices. J. Polym. Sci., Part B: Polym. Phys. 48, 2029-2037 (2010).

17 Tagaya, A., Harada, T. \& Koike, Y. Design of zero zero-birefringence polymers for injection molded products with low birefringence. Seikei-Kakou 21, 426-435 (2009) (in Japanese).

18 Ohkita, H., Ishibashi, K., Tsurumoto, D., Tagaya, A. \& Koike, Y. Compensation of the photoelastic birefringence of a polymer by doping with an anisotropic molecule. Appl. Phys. A. 81, 617-620 (2005).

19 Tagaya, A., Ohkita, H., Harada, T., Ishibashi, K. \& Koike, Y. Zero-birefringence optical polymers. Macromolecules 39, 3019-3023 (2006).

20 Kurashima, T., Tagaya, A. \& Koike, Y. in Digest of Technical Papers from the SID International Symposium 2011, May 15-20, 2011 Los Angeles, California 882-885 (The Society for Information Display, Campbell, CA, 2011).

21 Tagaya, A., Ohkita, H. \& Koike, Y. Zero-birefringence optical polymers by nanobirefringent crystals for liquid crystal displays. Mater. Sci. Eng., C 26, 966-970 (2006).

22 Shafiee, H., Shikama, K., Tagaya, A., Hardinghaus, F., Miyaji, T., Köhler, K. \& Koike, Y. Control of the birefringence dispersion of an optical polymer by doping with an inorganic crystal. Jpn. J. Appl. Phys. 50, 032602 (2011).

23 Uchiyama, A., Ishinabe, T., Miyashita, T., Uchida, T., Ono, Y. Y, \& Ikeda, Y. in IDW '04: Proceedings of the 11th International Display Workshops 647-650 (Society for Information Display, San Jose, CA, 2004).

24 Uchiyama, A. \& Yatabe, T. Control of wavelength dispersion of birefringence for oriented copolycarbonate films containing positive and negative birefringent units. Jpn. J. Appl. Phys. 42, 6941-6945 (2003).

25 Yamada, Y., Tagaya, A. \& Koike, Y. Birefringence Analysis of a Photonics Polymer Doped with a Birefringent Crystal. Proc. of SPIE 7213, 72130W-1-10 (2009).

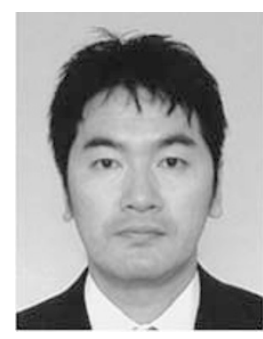

Akihiro Tagaya received BS (1991), MS (1993) and PhD (1996) degrees from Keio University. He worked for Hoechst Japan Limited from 1996 to 1998. He became a researcher of Prof. Yasuhiro Koike's Project conducted at Kanagawa Academy of Science and Technology in 1998. He joined ERATO and ERATO-SORST Koike Photonics Polymer Projects (JST) as a group leader from 2000 to 2011. He joined a FIRST program conducted by Prof. Koike as a Sub-theme leader from 2010 to present. He became an Instructor in 1999, an Assistant Professor in 2002, an Associate Professor in 2007 and a Project Professor in 2011 at Keio University. He became a Deputy Director at Keio Photonics Research Institute from 2011 to present. He received the SPSJ Hitachi Chemical Award in 2010, and Best Paper Award of 2009 by the Japan Society of Polymer Processing. His research interests include design of zero-zero-birefringence polymers for displays.

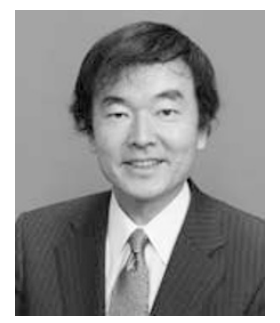

Yasuhiro Koike was born in Tokyo, Japan. He received his BS (1977), MS (1979), and PhD (1982) in applied chemistry from the Graduate School of Engineering of Keio University, Yokohama, Japan. He was a visiting researcher with AT\&T Bell Laboratories from 1989 to 1990. He has been Professor of Keio University since 1997. He is renowned for developing the high-bandwidth graded-index polymer optical fiber 'GI POF', highly scattered optical transmission polymer 'HSOT', zero-birefringence polymer, etc. He conducted the Koike Photonics Polymer Project in the ERATO-SORST program of Japan Science and Technology Agency (JST). His current project entitled 'Face-to-Face Communication Business by Ultra High-Speed Plastic Optical Fiber and High-Definition Photonics Polymer' has been selected as one of the FIRST programs of the Cabinet Office of Japan and its R\&Ds have been undertaken since March 2010. He received the International Engineering and Technology Award of the Society of Plastics Engineers in 1994, the Fujihara Award in 2001, and an honor of Medal with Purple Ribbon in Palace in 2006. In 2007, he was conferred an Honorary Doctorate from Eindhoven University of Technology. 